\title{
Design and Optimisation of a Simple Filter Group for Reactive Power Distribution
}

\author{
Ryszard Klempka \\ AGH University of Science and Technology, al. Mickiewicza 30, 30-059 Krakow, Poland \\ Correspondence should be addressed to Ryszard Klempka; klempka@agh.edu.pl
}

Received 27 June 2016; Revised 20 October 2016; Accepted 9 November 2016

Academic Editor: Riccardo Caponetto

Copyright (C) 2016 Ryszard Klempka. This is an open access article distributed under the Creative Commons Attribution License, which permits unrestricted use, distribution, and reproduction in any medium, provided the original work is properly cited.

Basic methods are presented to design a simple filter group and a method of shaping the resultant of the filter group's impedance characteristics (distribution of the characteristics' extremes) and then project equations were transformed into a uniform, common form that addresses issues of the reactive power distribution between component filters. The analysis also takes into account the filters' detuning from the reduced harmonics and quality factors of passive elements. Another important factor of the analysis considered was the power grid equivalent impedance affecting the filtration effectiveness. A criterion for the filter group's filtration effectiveness evaluation was proposed and optimisation was completed for the reactive power distribution between separate filters in the function of the power grid's equivalent inductance.

\section{Introduction}

The continuous development of civilization brings about a rapid increase in the number of nonlinear loads, causing unwanted reactive power in the power system and increased harmonics [1-4].

According to most theories, that is, Steinmetz, Buedanu, Fryze, Shepherd and Zakikhani, Depenbrock, Kusters and Moor, Page, Akagi, Nabaea, and Czarnecki to name those most famous, the nonlinear load is a source of unwanted reactive power and higher harmonics. In some of these theories, the discussion is around reactive power and imaginary power and in others the discussion is around passive current. This article is concerned with reactive power in the field of fundamental harmonics.

The simplest way to reduce reactive power and increased harmonics is to install passive-power filters. This solution is often applied for economic reasons.

There are two primary objectives in the application of filters for reactive power compensation. The first objective is concerned with the reduction of harmonics generated in the supply network. This objective can be achieved through filtering of the nonlinear loads to remove the harmonic components. The second objective is to compensate either the reactive power of the inductive load in the fundamental harmonic region or a reduction of the reactive power flowing in the mains. Inductive reactive power is the power associated with a phase shift between the fundamental components of the voltage and current (in this case, the voltage is phase shifted ahead of the current).

There are also many methods used to achieve reactive power compensation used throughout industry. The choice of a compensator is dependent on the nature of the compensated load and depends, for example, on the rate of change of reactive power. To achieve reactive power compensation, for example, to maintain a given power factor, voltage stabilization, and symmetrization network load, there is a need for compensation of the reactive power (compensation of fundamental harmonics and/or filtration of harmonics). The following are included within the most feasible technical implementation towards achieving this goal: synchronous machines with adjustable excitation current, batteries, capacitors, capacitor banks with serial reactors, passive filters and active higher harmonics (including STATCOM), and SVC (including TSC, FC/TCR, and TCR/TSC). However, because economic considerations also strongly influence the choices and because the latter are often relatively lower in price, 


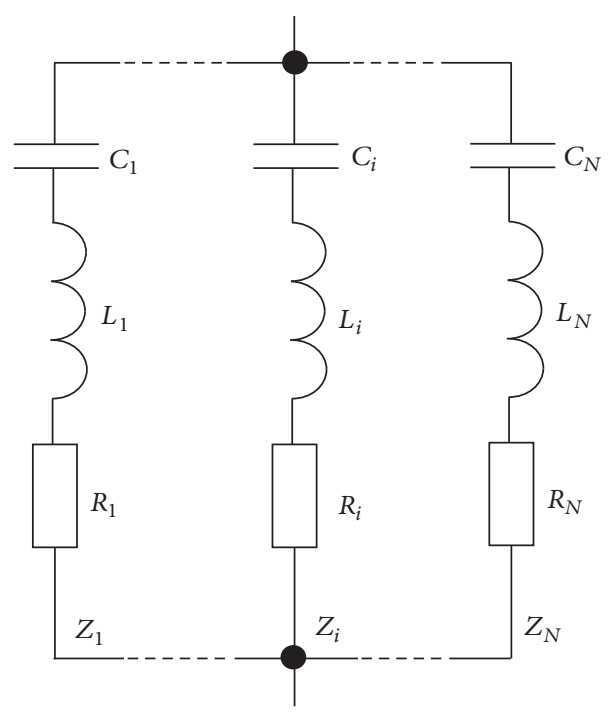

(a)

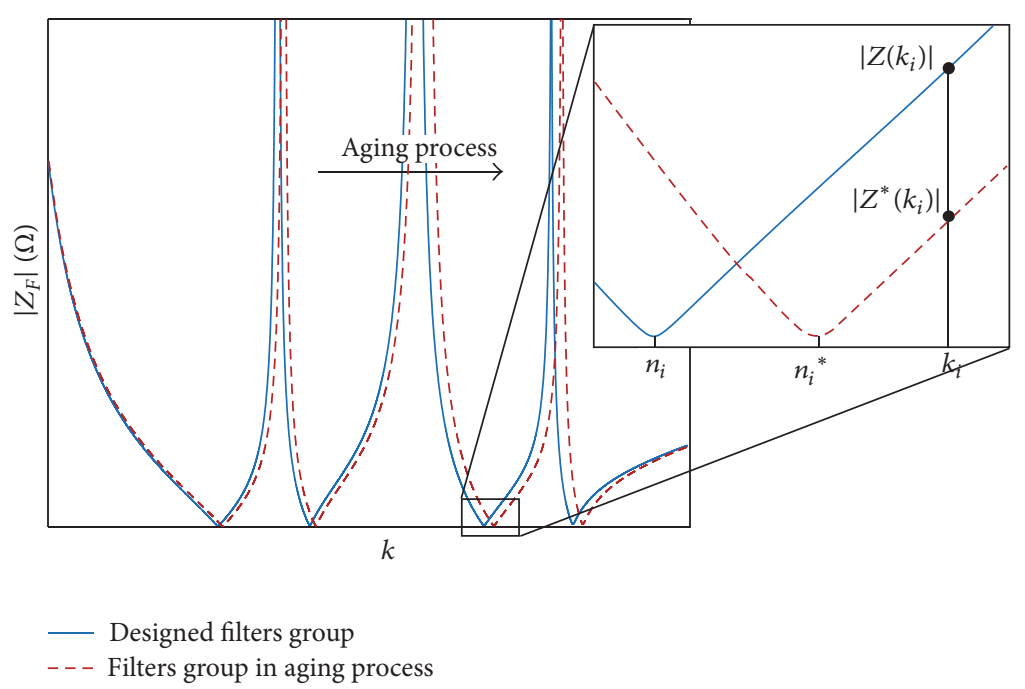

(b)

FIGURE 1: A simple filter group (a) diagram; (b) example of the projected characteristic impedance and the characteristics shifted to the aging filter elements. $n_{i}$, order harmonic of filter tuning; $n_{i}{ }^{*}$, order harmonic tuning of the filter shifted in the aging process; $k_{i}$, order of the reduced harmonic.

capacitor banks and filters are the solution most often used in industry.

There are many types of passive filters with different filtering properties; however, the basic structure is a singlebranch filter $[5,6]$.

Basic power-quality parameters are defined by standard EN 50160 [7]. It specifies, among other things, the maximumpermissible contents of voltage harmonics in the power system. In real industry installations, in order to meet the standard guidelines, there is often a need to apply several filters to reduce a higher number of harmonics. For this purpose, a group of simple filters connected in parallel can be designed (Figure 1).

Apart from the reduction of selected harmonics, the filter group's task is to reduce inductive reactive power, achieved by the capacitors. The basic task for the filter group designer is to make a decision about dividing the reduced reactive power into separate branches of the filter group.

The aim of this article is to demonstrate that classical methods of designing filter groups present in the literature can be summarised by the uniform issue of the reactive power distribution, which unifies these methods. The proposed method takes filters' detuning from the reduced harmonics and quality factors of passive elements into account. As the filter elements age, impedance characteristics move in the direction of higher frequencies. Only one item changes significantly over time in the filter structure: the capacitance of the capacitors. Inductance and the total resistance of the filter are subject mainly to temperature fluctuations (minor) and are relatively stable during the aging process. However, over a period of time, the capacitance has been observed to decrease. As a result, the tuning frequency of the filter (the resonance frequency of the serial filter) has a commensurate increase. To take this type of phenomena into account at the design stage, the filter is designed and tuned to a frequency slightly lower than the harmonic frequency it is intended to filter. As the filter shifts towards the harmonic frequency (as a result of the aging process), the filtration efficiency improves.

Then, a criterion was proposed to evaluate the filtration effectiveness of the whole group in order to compare methods. A significant role in the filtration effectiveness is played by the power grid equivalent impedance, which was also taken into account. A project example is presented, taking several variants into account in order to better evaluate the effect of the power grid equivalent inductance, and a case when it is necessary to increase the reduction of one of harmonics in order to meet the standard requirements is taken into account.

The measurement of equivalent impedance of the mains is to provide the power engineer with knowledge of circuit power at a given point in the supply network. While this value is subject to change, the power industry is able to determine the range of values of such changes. Therefore, when designing a filter group, the designer must calculate the minimum and maximum limits of short-circuit power. This calculation is necessary to avoid parallel resonances which may occur between the filter and the mains power so that the effectiveness of filtration can be assessed.

There are different methods of reducing reactive power and harmonics. Their advantages and disadvantages can be discussed. This article is limited only to the case when the designer has already made a decision about using a simple filter group and is then faced with the challenge of determining parameters of the component filters. The problem is dividing reactive power between filter branches so as to maximise the total reduction of selected harmonics. 


\section{Project Methods}

The main problem with designing simple group filters is reactive power distribution, which the whole group has to compensate between separate filtering branches. Below, five such methods will be presented. Each of them is based on two assumptions. The first assumption (different for each method) involves the decision about reactive power distribution and the second one (common for all methods) states that, for the filter resistances, $R_{i}=0$. This assumption is often made in literature because, due to low resistance values, they do not affect the location of the filter impedance characteristics' extremes, while resistances do affect, among other things, the filtration effectiveness and power losses in the filter; therefore, in the later part of the article, quality factors of passive elements will be taken into account.

The symbols used herein are the following:

$Q_{F}$, passive capacitive power, which is to be handled by the designed simple filter group

$U$, voltage at PCC

$\omega_{1}$, pulsation of the basic harmonic

$N$, the number of designed filters

$n_{i}$, orders of filter tuning harmonics

$Q_{i}$, passive capacitive power of the $i$ th filter

$C_{i}$, capacity of the $i$ th filter

$L_{i}$, inductance of the $i$ th filter

$R_{i}$, resistance of the $i$ th filter

$q_{L}$, choke quality factor

$q_{C}$, capacitor quality factor

$m_{i}$, orders of harmonics, where the maxima of the whole filter group impedance characteristics resultant are

Method A. Method A is based on the assumption that every group branch compensates the same reactive power [8]. Formulas (1) allow the designer to determine filter group parameters with such an assumption:

$$
\begin{aligned}
Q_{i} & =\frac{Q_{F}}{N} \\
C_{i} & =\frac{\left(1-n_{i}^{2}\right) \cdot Q_{F}}{N \cdot \omega_{1} \cdot n_{i}^{2} \cdot U^{2}} \\
L_{i} & =\frac{U^{2} \cdot N}{Q_{F} \cdot \omega_{1} \cdot\left(1-n_{i}^{2}\right)} .
\end{aligned}
$$

Method B. Method B is based on the assumption that the filter's reactive power is inversely proportional to the order of the harmonic to which the filter is tuned. From this assumption, formulas (2) are derived:

$$
\frac{Q_{i}}{Q_{i+1}}=\frac{n_{i+1}}{n_{i}} \Longrightarrow Q_{i}=\frac{Q_{F}}{n_{i} \cdot \sum_{j=1}^{N}\left(1 / n_{j}\right)}
$$

$$
\begin{aligned}
C_{i} & =\frac{\left(1-n_{i}^{2}\right) \cdot Q_{F}}{\omega_{1} \cdot n_{i}^{3} \cdot U^{2} \cdot \sum_{j=1}^{N}\left(1 / n_{j}\right)} \\
L_{i} & =\frac{n_{i} \cdot U^{2} \cdot \sum_{j=1}^{N}\left(1 / n_{j}\right)}{Q_{F} \cdot \omega_{1} \cdot\left(1-n_{i}^{2}\right)} .
\end{aligned}
$$

Method C. Method C is based on the assumption that the filter's reactive power is inversely proportional to the square of the order of the harmonic to which the filter is tuned. This assumption leads to the following formulas:

$$
\begin{aligned}
\frac{Q_{i}}{Q_{i+1}} & =\frac{n_{i+1}^{2}}{n_{i}^{2}} \Longrightarrow Q_{i}=\frac{Q_{F}}{n_{i}^{2} \cdot \sum_{j=1}^{N}\left(1 / n_{j}^{2}\right)} \\
C_{i} & =\frac{\left(1-n_{i}^{2}\right) \cdot Q_{F}}{\omega_{1} \cdot n_{i}^{4} \cdot U^{2} \cdot \sum_{j=1}^{N}\left(1 / n_{j}^{2}\right)} \\
L_{i} & =\frac{n_{i}^{2} \cdot U^{2} \cdot \sum_{j=1}^{N}\left(1 / n_{j}^{2}\right)}{Q_{F} \cdot \omega_{1} \cdot\left(1-n_{i}^{2}\right)} .
\end{aligned}
$$

Method D. The assumption in Method D is that all choke inductances in all group branches are the same. Formulas for this method are as follows:

$$
\begin{aligned}
Q_{i} & =\frac{Q_{F}}{\left(1-n_{i}^{2}\right) \cdot \sum_{j=1}^{N}\left(1 /\left(1-n_{j}^{2}\right)\right)} \\
C_{i} & =\frac{Q_{F}}{\omega_{1} \cdot n_{i}^{2} \cdot U^{2} \cdot \sum_{j=1}^{N}\left(1 /\left(1-n_{j}^{2}\right)\right)} \\
L_{i} & =L=\frac{U^{2} \cdot \sum_{j=1}^{N}\left(1 /\left(1-n_{j}^{2}\right)\right)}{Q_{F} \cdot \omega_{1}} .
\end{aligned}
$$

Method E. Method E is different from previous methods as, in all previous methods, some reactive power distribution is assumed and locations of the maxima of the filter group's impedance characteristics resultant come from this assumption. In this method, the opposite assumption is made. The location of the maxima of the characteristics' resultant $\left(m_{i}\right)$ is indicated, which results in reactive power distribution between separate filters. Such a presentation of the project task leads to formulation of the matrix equation $[9,10]$ :

$$
\left[\begin{array}{cccc}
\frac{n_{1}^{2} \cdot \omega_{1}}{n_{1}^{2}-1} & \frac{n_{2}^{2} \cdot \omega_{1}}{n_{2}^{2}-1} & \cdots & \frac{n_{N}^{2} \cdot \omega_{1}}{n_{N}^{2}-1} \\
\frac{n_{1}^{2} \cdot m_{1}}{n_{1}^{2}-m_{1}^{2}} & \frac{n_{2}^{2} \cdot m_{1}}{n_{2}^{2}-m_{1}^{2}} & \cdots & \frac{n_{N}^{2} \cdot m_{1}}{n_{N}^{2}-m_{1}^{2}} \\
\vdots & \vdots & \ddots & \vdots \\
\frac{n_{1}^{2} \cdot m_{N-1}}{n_{1}^{2}-m_{N-1}^{2}} & \frac{n_{2}^{2} \cdot m_{N-1}}{n_{2}^{2}-m_{N-1}^{2}} & \cdots & \frac{n_{N}^{2} \cdot m_{N-1}}{n_{N}^{2}-m_{N-1}^{2}}
\end{array}\right]\left[\begin{array}{c}
C_{1} \\
C_{2} \\
\vdots \\
C_{N}
\end{array}\right]
$$




$$
=\left[\begin{array}{c}
-\frac{Q_{F}}{U^{2}} \\
0 \\
\vdots \\
0
\end{array}\right]
$$

Method A. For Method A

$$
M_{i}=\frac{1}{N}
$$

Method B. For Method B

where

$$
1<n_{1}<m_{1}<n_{2}<m_{2} \cdots<m_{N-1}<n_{N}
$$

The solution of this matrix equation for specific numerical data can be obtained simply, for example, via the Matlab package. However, the general analytical solution of this matrix equation will be needed for the purposes of this article:

$$
\begin{aligned}
C_{i}= & \frac{-Q_{F}}{\omega_{1} \cdot U^{2}} \cdot \frac{\prod_{j=1}^{N}\left(n_{j}^{2}-1\right)}{\prod_{j=1}^{N-1}\left(m_{j}^{2}-1\right)} \\
& \cdot \frac{\prod_{j=1}^{N-1}\left(n_{i}^{2}-m_{j}^{2}\right)}{n_{i}^{2} \cdot \prod_{j=1, i \neq j}^{N}\left(n_{i}^{2}-n_{j}^{2}\right)} .
\end{aligned}
$$

Formulas enabling the designer to determine values of inductive and reactive powers of separate branches are specified by the following formulas:

$$
\begin{aligned}
L_{i} & =\frac{-U^{2}}{\omega_{1} \cdot Q_{F}} \cdot \frac{\prod_{j=1}^{N-1}\left(m_{j}^{2}-1\right)}{\prod_{j=1}^{N}\left(n_{j}^{2}-1\right)} \cdot \frac{\prod_{j=1, i \neq j}^{N}\left(n_{i}^{2}-n_{j}^{2}\right)}{\prod_{j=1}^{N-1}\left(n_{i}^{2}-m_{j}^{2}\right)} \\
Q_{i} & =\frac{-Q_{F}}{1-n_{i}^{2}} \cdot \frac{\prod_{j=1}^{N}\left(n_{j}^{2}-1\right)}{\prod_{j=1}^{N-1}\left(m_{j}^{2}-1\right)} \cdot \frac{\prod_{j=1}^{N-1}\left(n_{i}^{2}-m_{j}^{2}\right)}{n_{i}^{2} \cdot \prod_{j=1, i \neq j}^{N}\left(n_{i}^{2}-n_{j}^{2}\right)} .
\end{aligned}
$$

Equation (9) is true for every of the above methods:

$$
Q_{F}=\sum_{i=1}^{N} Q_{i}
$$

The above methods (A-D) are most often applied when designing filter groups. The matrix method (E) is the interesting alternative for previous methods, enabling the designer to consciously design the shape of the filter group's impedance characteristics.

The issue of shifting the impedance characteristics maxima was discussed in [11] through the selection of the filtering system's transmittance coefficients in order to avoid resonance for harmonics coming from the power grid.

\section{The Analysis of Project Methods}

Analysing formulas for capacitances, inductances, and reactive powers for the $i$ th filter in the group for the five presented methods, the possibility to introduce a simplification can be seen:

$$
B=\frac{U^{2}}{\omega_{1} \cdot Q_{F}}
$$

and coefficients $M_{i}$ can be defined for every method:

$$
M_{i}=\frac{1}{n_{i} \cdot \sum_{j=1}^{N}\left(1 / n_{j}\right)} .
$$

Method C. For Method C

$$
M_{i}=\frac{1}{n_{i}^{2} \cdot \sum_{j=1}^{N}\left(1 / n_{j}^{2}\right)} .
$$

Method D. For Method D

$$
M_{i}=\frac{1}{\left(1-n_{i}^{2}\right) \cdot \sum_{j=1}^{N}\left(1 /\left(1-n_{j}^{2}\right)\right)} .
$$

Method E. For Method E

$$
\begin{aligned}
M_{i}= & \frac{-1}{\left(1-n_{i}^{2}\right)} \cdot \frac{\prod_{j=1}^{N}\left(n_{j}^{2}-1\right)}{\prod_{j=1}^{N-1}\left(m_{j}^{2}-1\right)} \\
& \cdot \frac{\prod_{j=1}^{N-1}\left(n_{i}^{2}-m_{j}^{2}\right)}{n_{i}^{2} \cdot \prod_{j=1, i \neq j}^{N}\left(n_{i}^{2}-n_{j}^{2}\right)} .
\end{aligned}
$$

Introducing (10)-(15) to (1)-(4) and (7)-(8), we obtain general formulas true for all methods:

$$
\begin{aligned}
C_{i} & =\frac{M_{i} \cdot\left(1-n_{i}^{2}\right)}{B \cdot n_{i}^{2} \cdot \omega_{1}^{2}} \\
L_{i} & =\frac{B}{M_{i} \cdot\left(1-n_{i}^{2}\right)} \\
Q_{i} & =Q_{F} \cdot M_{i} .
\end{aligned}
$$

From (18), it can be observed that the coefficients $M_{i}$ determine what part of the total reactive power falls on the ith filter in the group. Thus, (19) is true:

$$
\sum_{i=1}^{N} M_{i}=1
$$

where $M_{i}$ is the part of the total reactive power compensated by $i$ th filter (reactive power distribution coefficient).

Thanks to coefficients $M_{i}$, it is possible to convert the filter parameters determined by one of the presented methods into values, which we would obtain by converting by another method:

$$
\begin{gathered}
\frac{C_{i}^{\mathrm{I}}}{C_{i}^{\mathrm{II}}}=\frac{M_{i}^{\mathrm{I}}}{M_{i}^{\mathrm{II}}} ; \\
\frac{L_{i}^{\mathrm{I}}}{L_{i}^{\mathrm{II}}}=\frac{M_{i}^{\mathrm{II}}}{M_{i}^{\mathrm{I}}} ; \\
\frac{Q_{i}^{\mathrm{I}}}{Q_{i}^{\mathrm{II}}}=\frac{M_{i}^{\mathrm{I}}}{M_{i}^{\mathrm{II}}},
\end{gathered}
$$


where I index denotes any one method and II index denotes any second method.

The impedance of a single filter for the $k$ th harmonic is equal to

$$
Z_{i}(k)=j \cdot \frac{B \cdot \omega_{1} \cdot\left(k^{2}-n_{i}^{2}\right)}{M_{i} \cdot\left(1-n_{i}^{2}\right) \cdot k}
$$

and impedance of the whole filter group

$$
Z(k)=j \cdot \frac{B \cdot \omega_{1}}{k \cdot \sum_{i=1}^{N} M_{i} \cdot\left(1-n_{i}^{2}\right) /\left(k^{2}-n_{i}^{2}\right)} .
$$

The impedance characteristics' minima specify values $n_{i}$, while the location of the maxima is determined by square roots $m_{j}$ of the following equation:

$$
0=\sum_{i=1}^{N} \frac{M_{i} \cdot\left(1-n_{i}^{2}\right)}{m_{j}^{2}-n_{i}^{2}}
$$

To date, the analysis of the single-branch filter group was done assuming the lack of the filter's resistance part. This was possible due to the lack of an effect or due to a marginal effect of filter resistance on the location of the impedance characteristics' extrema. Resistance, however, has a significant effect on the value of impedance in the characteristics' extrema. The main components causing the existence of filter resistance are its passive elements. Chokes and capacitors are characterised through their quality factor $[12,13]$. Depending on the elements' power and filters' operating voltage, typical values of quality factors of chokes (24) and capacitors (25) are specified:

$$
\begin{aligned}
& q_{L}=\frac{\omega_{1} \cdot L_{i}}{R_{L i}} \\
& q_{C}=\frac{1}{\omega_{1} \cdot C_{i} \cdot R_{C i}} .
\end{aligned}
$$

The resistance of $i$ th filter is equal to

$$
R_{i}=R_{L i}+R_{C i}
$$

Using formulas (16), (17), (24), and (25), the filter resistance is specified by the following equation:

$$
R_{i}=\frac{B \cdot \omega_{1} \cdot\left(q_{C}+q_{L} \cdot n_{i}^{2}\right)}{M_{i} \cdot\left(1-n_{i}^{2}\right) \cdot q_{L} \cdot q_{C}} .
$$

Similarly, as in the case of reactive power, capacitance, and inductance and also for the resistance of a single filter, the conversion of value between methods is possible:

$$
\frac{R_{i}^{\mathrm{I}}}{R_{i}^{\mathrm{II}}}=\frac{M_{i}^{\mathrm{II}}}{M_{i}^{\mathrm{I}}} .
$$

Capacitances and reactive powers of filters are directly proportional to reactive power distribution coefficients $M_{i}$, and inductance and resistance are inversely proportional to these coefficients.

The impedance of a single filter for the $k$ th harmonic is equal to

$$
\begin{aligned}
Z_{i}(k) & \\
= & \frac{B \cdot \omega_{1}}{M_{i} \cdot\left(1-n_{i}^{2}\right) \cdot q_{L} \cdot q_{C} \cdot k} \\
& \cdot\left[k \cdot\left(q_{C}+q_{L} \cdot n_{i}^{2}\right)+j \cdot\left(k^{2}-n_{i}^{2}\right) \cdot q_{L} \cdot q_{C}\right] .
\end{aligned}
$$

It is worth noting that, due to aging of filter elements and thus shifting of the impedance characteristics' resultant to the right, filters become detuned from harmonics which they are to reduce to the slightly decreased frequency. This detuning, depending on the reduced harmonic, fluctuates from $2 \%$ to $10 \%$ below the reduced harmonic [1]. Studying (29) for a case when the filter is detuned from harmonic $k$ to order $n_{i}$, a real part of impedance dependent on the elements' quality factors and an imaginary part resulting from detuning can be noticed. While we have no influence on the real part of the impedance, a decrease in detuning from the reduced harmonic decreases the filter impedance, and thus the filtration effectiveness increases.

As it can be easily demonstrated, (30) is true:

$$
\frac{\operatorname{Re}\left\{Z_{i}^{\mathrm{I}}(k)\right\}}{\operatorname{Re}\left\{Z_{i}^{\mathrm{II}}(k)\right\}}=\frac{\operatorname{Im}\left\{Z_{i}^{\mathrm{I}}(k)\right\}}{\operatorname{Im}\left\{Z_{i}^{\mathrm{II}}(k)\right\}}=\frac{Z_{i}^{\mathrm{I}}(k)}{Z_{i}^{\mathrm{II}}(k)}=\frac{M_{i}^{\mathrm{II}}}{M_{i}^{\mathrm{I}}} .
$$

Impedance of the whole filter group is specified by the following equation:

$$
Z(k)=\frac{B \cdot \omega_{1}}{q_{\mathrm{L}} \cdot q_{\mathrm{C}} \cdot k \cdot \sum_{i=1}^{N}\left(M_{i} \cdot\left(1-n_{i}^{2}\right) /\left(k \cdot\left(q_{C}+q_{L} \cdot n_{i}^{2}\right)+j \cdot\left(k^{2}-n_{i}^{2}\right) \cdot q_{L} \cdot q_{C}\right)\right)}
$$

The filter group's impedance has a significant influence on the filtration effectiveness: the lower the filter impedance for the reduced harmonics, the higher the reduction of these harmonics. Another important factor affecting the harmonic 
reduction is the power grid equivalent impedance for these harmonics:

$$
\begin{aligned}
& Z_{S}(k)=R_{S}+j \cdot \omega_{1} \cdot k \cdot L_{S} \\
& \varepsilon(k)=1-\left\|\frac{Z(k)}{Z(k)+Z_{S}(k)}\right\| .
\end{aligned}
$$

This coefficient determines which harmonic part will be reduced. Value 1 denotes the total harmonic reduction, whereas value 0 means no reduction, and negative values denote $k$ th harmonic amplification.

The evaluated filtration effectiveness of the selected harmonic concerns the whole filter group, not a single filter. Every filter in the group affects the filtration effectiveness of every harmonic:

$$
\varepsilon(k)=1-\left\|\frac{B \cdot \omega_{1}}{B \cdot \omega_{1}+\left(R_{S}+j \cdot \omega_{1} \cdot k \cdot L_{S}\right) \cdot q_{L} \cdot q_{C} \cdot k \cdot \sum_{i=1}^{N}\left(M_{i} \cdot\left(1-n_{i}^{2}\right) /\left(k \cdot\left(q_{C}+q_{L} \cdot n_{i}^{2}\right)+j \cdot\left(k^{2}-n_{i}^{2}\right) \cdot q_{L} \cdot q_{C}\right)\right)}\right\|
$$

It is worth noting that all parameters in formula (33) are constant parameters of the project task except coefficients $M_{i}$. We can influence the filtration effectiveness of harmonics by the total reactive power distribution between single filters. It must be noted that the number of designed filters is $N$, and the effectiveness improvement of one of them can deteriorate other filters' filtration effectiveness.

In order to compare methods of simple filter groups' designed, the quality coefficient (34), which shows the summary reduction of selected harmonics by the whole group, is determined:

$$
\begin{aligned}
\varepsilon_{S} & =\sum_{j=1}^{N} \varepsilon\left(k_{j}\right) \\
\varepsilon_{S} & =N \\
& -\sum_{j=1}^{N}\left\|\frac{B \cdot \omega_{1}}{B \cdot \omega_{1}+\left(R_{S}+j \cdot \omega_{1} \cdot k_{j} \cdot L_{S}\right) \cdot q_{L} \cdot q_{C} \cdot k_{j} \cdot \sum_{i=1}^{N}\left(M_{i} \cdot\left(1-n_{i}^{2}\right) /\left(k_{j} \cdot\left(q_{C}+q_{L} \cdot n_{i}^{2}\right)+j \cdot\left(k_{j}^{2}-n_{i}^{2}\right) \cdot q_{L} \cdot q_{C}\right)\right)}\right\|,
\end{aligned}
$$

where $k_{j}$ are orders of harmonics that are to be reduced by the filter group.

Criterion (35) shows the summary effectiveness of filtration of harmonics that are to be reduced by the whole group. The power grid equivalent impedance significantly affects the value of this coefficient.

Equation (35) can easily be extended to a greater number of harmonics and not only to harmonics to which this group was designed. That is, the effectiveness of filtration of a greater number of harmonics by the designed filter group can be evaluated. In the later part of this article, the filtration effectiveness of group tuning harmonics will be evaluated.

In order to evaluate this influence, a calculation example of the design of four simple filter groups cooperating with a 6-pulse converter will be presented.

\section{Project Example}

As an example illustrating the use of the above equations, the project task of the group with 4 simple filters with a total reactive power of $1 \mathrm{Mvar}$, per voltage $6 \mathrm{kV}$, for the reduction of harmonics $5,7,11$, and $13\left(n_{i}=4.7,6.7,10.5,12.5\right)$, will be presented. For the matrix method, the impedance characteristics' maxima should fall on harmonics of orders 5.7, 8.6, and 11.5. The quality factor of the elements is $q_{C}=$ 5000 and $q_{L}=100$. The filter group design takes two cases of the power grid, with the following parameters, into account:

$$
\begin{aligned}
& \left(\mathrm{S}_{1}\right) R_{S}=100 \mathrm{~m} \Omega \text { and } L_{S}=3 \mathrm{mH}(\text { case } \mathrm{F}) . \\
& \left(\mathrm{S}_{2}\right) R_{S}=30 \mathrm{~m} \Omega \text { and } L_{S}=1 \mathrm{mH}(\text { case } \mathrm{G}) .
\end{aligned}
$$

Table 1 contains a summary of parameters of filter groups designed by the five presented methods for two different power grid equivalent impedances.

The values of impedance characteristics' minima depend on the filters' resistance. The impedances of the filter group for the eliminated harmonics depend strongly on filters' detuning and, to a small degree, on their resistance.

The reduction of selected harmonics depends on the given filter's reactive power: the greater the power, the higher the filtration effectiveness. Method A demonstrates the lowest reduction of the 5th harmonic and methods $\mathrm{C}, \mathrm{D}$, and $\mathrm{E}$ demonstrate the highest reduction of this harmonic.

The power grid equivalent impedance affects greatly the filter group's filtration effectiveness. Along with the increase in equivalent impedance, the filtration effectiveness increases. 
TABLE 1: Basic parameters of filter groups designed by different methods.

\begin{tabular}{|c|c|c|c|c|c|c|}
\hline \multirow{2}{*}{ Size } & & \multicolumn{5}{|c|}{ Method } \\
\hline & & $\mathrm{A}$ & $\mathrm{B}$ & $\mathrm{C}$ & $\mathrm{D}$ & $\mathrm{E}$ \\
\hline$M_{1}$ & & 0.25 & 0.3960 & 0.5453 & 0.5527 & 0.5440 \\
\hline$M_{2}$ & & 0.25 & 0.2778 & 0.2683 & 0.2656 & 0.2251 \\
\hline$M_{3}$ & & 0.25 & 0.1773 & 0.1093 & 0.1067 & 0.1116 \\
\hline$M_{4}$ & & 0.25 & 0.1489 & 0.0771 & 0.0751 & 0.1193 \\
\hline$Q_{1}$ & kvar & -250 & -396.02 & -545.31 & -552.67 & -544.01 \\
\hline$Q_{2}$ & kvar & -250 & -277.81 & -268.34 & -265.57 & -225.10 \\
\hline$Q_{3}$ & kvar & -250 & -177.27 & -109.26 & -106.69 & -111.62 \\
\hline$Q_{4}$ & kvar & -250 & -148.90 & -77.09 & -75.08 & -119.27 \\
\hline$C_{1}$ & $\mu \mathrm{F}$ & 21.10 & 33.43 & 46.03 & 46.65 & 45.92 \\
\hline $\mathrm{C}_{2}$ & $\mu \mathrm{F}$ & 21.61 & 24.02 & 23.20 & 22.96 & 19.46 \\
\hline$C_{3}$ & $\mu \mathrm{F}$ & 21.90 & 15.53 & 9.57 & 9.35 & 9.78 \\
\hline$C_{4}$ & $\mu \mathrm{F}$ & 21.96 & 13.08 & 6.77 & 6.60 & 10.48 \\
\hline$L_{1}$ & $\mathrm{mH}$ & 21.73 & 13.72 & 9.96 & 9.83 & 9.99 \\
\hline$L_{2}$ & $\mathrm{mH}$ & 10.44 & 9.40 & 9.73 & 9.83 & 11.60 \\
\hline$L_{3}$ & $\mathrm{mH}$ & 4.20 & 5.92 & 9.60 & 9.83 & 9.40 \\
\hline$L_{4}$ & $\mathrm{mH}$ & 2.95 & 4.96 & 9.57 & 9.83 & 6.19 \\
\hline$m_{1}$ & & 5.14 & 5.39 & 5.66 & 5.67 & 5.7 \\
\hline$m_{2}$ & & 7.67 & 8.23 & 8.91 & 8.93 & 8.6 \\
\hline$m_{3}$ & & 11.43 & 11.57 & 11.75 & 11.75 & 11.5 \\
\hline$R_{1}$ & $\mathrm{~m} \Omega$ & 98.44 & 62.15 & 45.13 & 44.53 & 45.24 \\
\hline$R_{2}$ & $\mathrm{~m} \Omega$ & 62.27 & 56.03 & 58.01 & 58.62 & 69.15 \\
\hline$R_{3}$ & $\mathrm{~m} \Omega$ & 42.24 & 59.58 & 96.66 & 98.99 & 94.62 \\
\hline$R_{4}$ & $\mathrm{~m} \Omega$ & 38.26 & 64.24 & 124.07 & 127.41 & 80.20 \\
\hline$Z_{1}(5)$ & $\Omega$ & $0.098+j 3.974$ & $0.062+j 2.509$ & $0.045+j 1.822$ & $0.045+j 1.798$ & $0.045+j 1.826$ \\
\hline$Z_{2}(7)$ & $\Omega$ & $0.062+j 1.926$ & $0.056+j 1.734$ & $0.058+j 1.795$ & $0.059+j 1.814$ & $0.069+j 2.140$ \\
\hline$Z_{3}(11)$ & $\Omega$ & $0.042+j 1.288$ & $0.060+j 1.817$ & $0.097+j 2.947$ & $0.099+j 3.018$ & $0.095+j 2.885$ \\
\hline$Z_{4}(13)$ & $\Omega$ & $0.038+j 0.910$ & $0.064+j 1.527$ & $0.124+j 2.950$ & $0.127+j 3.029$ & $0.080+j 1.907$ \\
\hline$Z(1)$ & $\Omega$ & $0.0151-j 36$ & $0.0174-j 36$ & $0.0195-j 36$ & $0.0196-j 36$ & $0.0192-j 36$ \\
\hline$Z(5)$ & $\Omega$ & $0.841+j 11.13$ & $0.157+j 3.881$ & $0.074+j 2.301$ & $0.072+j 2.254$ & $0.072+j 2.277$ \\
\hline$Z(7)$ & $\Omega$ & $0.109+j 2.498$ & $0.067+j 1.873$ & $0.055+j 1.722$ & $0.055+j 1.732$ & $0.069+j 2.092$ \\
\hline$Z(11)$ & $\Omega$ & $0.120+j 1.995$ & $0.115+j 2.351$ & $0.125+j 3.140$ & $0.126+j 3.193$ & $0.199+j 3.823$ \\
\hline$Z(13)$ & $\Omega$ & $0.028+j 0.762$ & $0.041+j 1.205$ & $0.066+j 2.106$ & $0.067+j 2.154$ & $0.053+j 1.524$ \\
\hline \multicolumn{7}{|c|}{$S_{1}$} \\
\hline$\varepsilon(5)$ & & 0.297 & 0.548 & 0.672 & 0.676 & 0.674 \\
\hline$\varepsilon(7)$ & & 0.725 & 0.779 & 0.793 & 0.792 & 0.759 \\
\hline$\varepsilon(11)$ & & 0.838 & 0.815 & 0.767 & 0.764 & 0.730 \\
\hline $\mathcal{E}(13)$ & & 0.941 & 0.910 & 0.853 & 0.850 & 0.889 \\
\hline$\varepsilon_{S}$ & & 2.802 & 3.052 & 3.086 & 3.083 & 3.053 \\
\hline \multicolumn{7}{|c|}{$S_{2}$} \\
\hline$\varepsilon(5)$ & & 0.123 & 0.288 & 0.406 & 0.411 & 0.408 \\
\hline$\varepsilon(7)$ & & 0.468 & 0.540 & 0.561 & 0.559 & 0.512 \\
\hline$\varepsilon(11)$ & & 0.634 & 0.595 & 0.524 & 0.520 & 0.474 \\
\hline$\varepsilon(13)$ & & 0.843 & 0.772 & 0.660 & 0.655 & 0.728 \\
\hline$\underline{\varepsilon_{S}}$ & & 2.067 & 2.195 & 2.150 & 2.144 & 2.123 \\
\hline
\end{tabular}




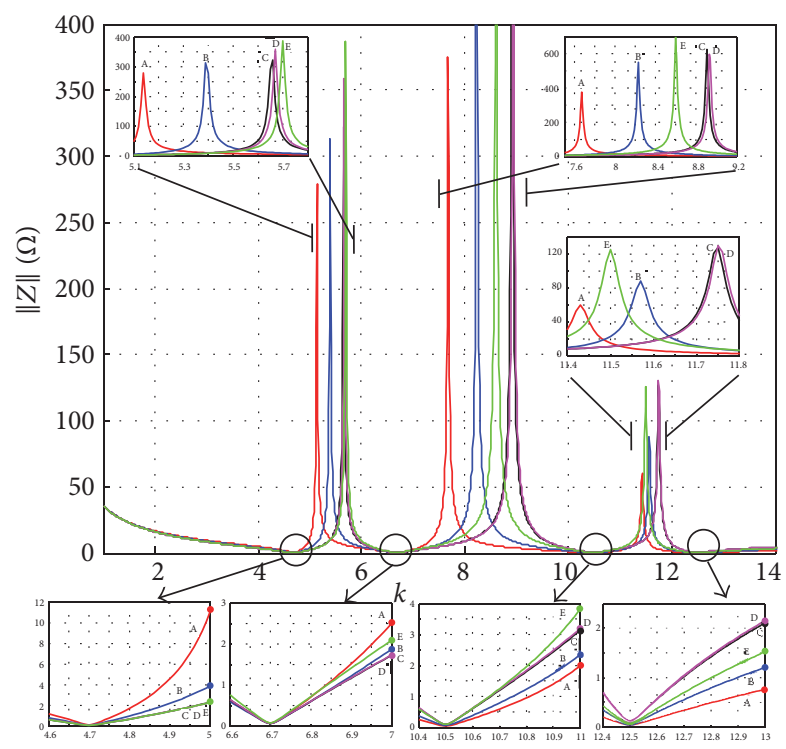

FIGURE 2: Impedance characteristics of the filter groups designed by methods A, B, C, D, and E with enlarged fragments characteristic of these groups.

Figure 2 presents impedance characteristics' resultants for the filter groups designed by different methods. Fragments of characteristics, where their extrema locations are, are enlarged in the graph.

The summary reduction coefficient for grid $S_{1}$ is the highest for Method $\mathrm{C}$, and for grid $S_{2}$ it is highest for Method B. Hence, the conclusion is that the selection of this design method should depend on the power grid equivalent impedance. Additionally, it should be decided whether the selected design method guarantees the best possible harmonic reduction. In the next chapter, the optimisation of reactive power distribution between separate filters will be performed in order to ensure the maximum reduction of selected harmonics, that is, the maximisation of coefficient (35) for different values of the power grid equivalent impedance.

\section{The Filter Effectiveness Optimisation}

Many authors in the scientific community have considered the question of optimal filter selection. They try to answer this question in different ways. They specify different optimality criteria and apply different optimisation tools $[1,6,14-18]$ including methods of artificial intelligence [19-24].

The results presented in the previous chapter suggest that, through the selection of reactive power distribution $M_{i}$ between simple filters, it is possible to influence the whole group's filtration effectiveness. There are as many coefficients $M_{i}$ as there are filters $(N)$. The selection of coefficients $M_{i}$ is tantamount to the placement of impedance characteristics' maxima, which are $N-1$, that is, by one parameter fewer to optimise. Thanks to (6), it is possible to determine the acceptable range of variation of every value $m_{i}$ (maxima locations). For the optimal maxima locations, using (15), the values of the coefficients $M_{i}$ are determined, and, later, from (35), the filtration effectiveness of the designed group is determined.

Another way to determine reactive power distribution on the basis of optimal maxima locations is to use (23) and (19), from which a system of equations is built in matrix form:

$$
\begin{aligned}
& {\left[\begin{array}{cccc}
1 & 1 & \cdots & 1 \\
\frac{1-n_{1}^{2}}{m_{1}^{2}-n_{1}^{2}} & \frac{1-n_{2}^{2}}{m_{1}^{2}-n_{2}^{2}} & \cdots & \frac{1-n_{N}^{2}}{m_{1}^{2}-n_{N}^{2}} \\
\frac{1-n_{1}^{2}}{m_{2}^{2}-n_{1}^{2}} & \frac{1-n_{2}^{2}}{m_{2}^{2}-n_{2}^{2}} & \cdots & \frac{1-n_{N}^{2}}{m_{2}^{2}-n_{N}^{2}} \\
\vdots & \vdots & \ddots & \vdots \\
\frac{1-n_{1}^{2}}{m_{N-1}^{2}-n_{1}^{2}} & \frac{1-n_{2}^{2}}{m_{N-1}^{2}-n_{2}^{2}} & \cdots & \frac{1-n_{N}^{2}}{m_{N-1}^{2}-n_{N}^{2}}
\end{array}\right]\left[\begin{array}{c}
M_{1} \\
M_{2} \\
\vdots \\
M_{N-1} \\
M_{N}
\end{array}\right]} \\
& =\left[\begin{array}{c}
1 \\
0 \\
\vdots \\
0 \\
0
\end{array}\right] .
\end{aligned}
$$

Optimisations of the impedance characteristics' maxima distribution will be performed for both grids $S_{1}$ and $S_{2}$ without limitations and for grid $S_{2}$ with two different limitations. The limitation in the performed optimisation means that the degree of the selected harmonic reduction by the whole filter group must be higher or equal to the set value in order to meet the standard requirements:
(a) $\varepsilon(5) \geq 0.45$ (case $\mathrm{H})$.
(b) $\varepsilon(5) \geq 0.50$ (case I).

Due to the use of the Matlab package and function fminsearch, which is based on the Nelder-Mead method for minimisation, the criterion for the performed optimisation will have the form in (37) for cases without limitations and that in (38) for cases with a limitation:

$$
\begin{aligned}
& y=\frac{1}{\varepsilon_{S}} \\
& y= \begin{cases}\frac{1}{\varepsilon_{S}} \\
\frac{(1+\operatorname{limit}-\varepsilon(5))^{2}}{\varepsilon_{S}} & \text { dla } \varepsilon(5)<\text { limit. }\end{cases}
\end{aligned}
$$

Table 2 contains a summary of basic parameters of optimised filter groups for the power grid with different equivalent impedances and by taking the assumed reduction of the 5th harmonics into account. For grid $S_{1}$, coefficient $M_{1}$ is lower compared to Methods C, D, and E. For grid $S_{2}$ with lower equivalent impedance, coefficient $M_{1}$ is approximate to Method B; taking the limitation into account causes an increase in coefficient $M_{1}$.

Values of the coefficient $M_{2}$ are approximate to each other, except for the cases of grid $S_{2}$ with limitations; the 
TABLE 2: Basic parameters of optimised filter groups for different power grid equivalent impedance and by taking possible design limitations in the form of the assumed reduction of the selected harmonic into account.

\begin{tabular}{|c|c|c|c|c|c|}
\hline \multirow{3}{*}{ Size } & & $F^{*}$ & $G^{*}$ & $H^{*}$ & $I^{*}$ \\
\hline & & $S_{1}$ & $S_{2}$ & $S_{2}$ & $S_{2}$ \\
\hline & & \multicolumn{2}{|c|}{ Without limitations } & $\varepsilon(5) \geq 0.45$ & $\varepsilon(5) \geq 0.50$ \\
\hline$M_{1}$ & & 0.5110 & 0.4135 & 0.6065 & 0.6990 \\
\hline$M_{2}$ & & 0.2391 & 0.2667 & 0.1584 & 0.1088 \\
\hline$M_{3}$ & & 0.1829 & 0.2342 & 0.1721 & 0.1418 \\
\hline$M_{4}$ & & 0.0669 & 0.0856 & 0.0630 & 0.0504 \\
\hline$Q_{1}$ & kvar & -511.0 & -413.5 & -606.5 & -699.0 \\
\hline$Q_{2}$ & kvar & -239.1 & -266.7 & -158.4 & -108.8 \\
\hline$Q_{3}$ & kvar & -182.9 & -234.2 & -172.1 & -141.8 \\
\hline$Q_{4}$ & kvar & -66.9 & -85.6 & -63.0 & -50.4 \\
\hline$C_{1}$ & $\mu \mathrm{F}$ & 43.14 & 34.91 & 51.20 & 59.01 \\
\hline $\mathrm{C}_{2}$ & $\mu \mathrm{F}$ & 20.67 & 23.06 & 13.69 & 9.41 \\
\hline$C_{3}$ & $\mu \mathrm{F}$ & 16.03 & 20.52 & 15.08 & 12.42 \\
\hline $\mathrm{C}_{4}$ & $\mu \mathrm{F}$ & 5.88 & 7.52 & 5.53 & 4.43 \\
\hline$L_{1}$ & $\mathrm{mH}$ & 10.6 & 13.1 & 9.0 & 7.8 \\
\hline$L_{2}$ & $\mathrm{mH}$ & 10.9 & 9.8 & 16.5 & 24.0 \\
\hline$L_{3}$ & $\mathrm{mH}$ & 5.7 & 4.5 & 6.1 & 7.4 \\
\hline$L_{4}$ & $\mathrm{mH}$ & 11.0 & 8.6 & 11.7 & 14.6 \\
\hline$m_{1}$ & & 5.6244 & 5.4290 & 5.8922 & 6.1402 \\
\hline$m_{2}$ & & 8.4113 & 8.1620 & 8.2929 & 8.4211 \\
\hline$m_{3}$ & & 11.9699 & 11.9457 & 11.9649 & 11.9936 \\
\hline$R_{1}$ & $\mathrm{~m} \Omega$ & 48.2 & 59.5 & 40.6 & 35.2 \\
\hline$R_{2}$ & $\mathrm{~m} \Omega$ & 65.1 & 58.4 & 98.3 & 143.1 \\
\hline$R_{3}$ & $\mathrm{~m} \Omega$ & 57.7 & 45.1 & 61.4 & 74.5 \\
\hline$R_{4}$ & $\mathrm{~m} \Omega$ & 143 & 111.70 & 151.8 & 189.8 \\
\hline$Z_{1}(5)$ & $\Omega$ & $0.048+j 1.944$ & $0.060+j 2.403$ & $0.041+j 1.638$ & $0.035+j 1.421$ \\
\hline$Z_{2}(7)$ & $\Omega$ & $0.065+j 2.014$ & $0.058+j 1.806$ & $0.098+j 3.040$ & $0.143+j 4.426$ \\
\hline$Z_{3}(11)$ & $\Omega$ & $0.058+j 1.761$ & $0.045+j 1.375$ & $0.061+j 1.871$ & $0.075+j 2.271$ \\
\hline$Z_{4}(13)$ & $\Omega$ & $0.143+j 3.400$ & $0.112+j 2.657$ & $0.152+j 3.610$ & $0.190+j 4.512$ \\
\hline$Z(1)$ & $\Omega$ & $0.019-j 36$ & $0.018-j 36$ & $0.020-j 36$ & $0.021-j 36$ \\
\hline$Z(5)$ & $\Omega$ & $0.083+j 2.515$ & $0.139+j 3.586$ & $0.057+j 1.919$ & $0.044+j 1.571$ \\
\hline$Z(7)$ & $\Omega$ & $0.069+j 2.032$ & $0.071+j 1.955$ & $0.095+j 2.908$ & $0.109+j 3.717$ \\
\hline$Z(11)$ & $\Omega$ & $0.065+j 1.807$ & $0.054+j 1.457$ & $0.070+j 1.934$ & $0.081+j 2.287$ \\
\hline$Z(13)$ & $\Omega$ & $0.059+j 2.110$ & $0.049+j 1.698$ & $0.064+j 2.252$ & $0.075+j 2.726$ \\
\hline$\varepsilon(5)$ & & 0.6520 & 0.3045 & 0.4500 & 0.5000 \\
\hline$\varepsilon(7)$ & & 0.7645 & 0.5293 & 0.4305 & 0.3716 \\
\hline$\varepsilon(11)$ & & 0.8515 & 0.7032 & 0.6410 & 0.6016 \\
\hline$\varepsilon(13)$ & & 0.8530 & 0.7062 & 0.6446 & 0.5996 \\
\hline$\varepsilon_{S}$ & & 3.1210 & 2.2432 & 2.1661 & 2.0730 \\
\hline
\end{tabular}

${ }^{*} F$, power grid with parameters: $R_{S}=100 \mathrm{~m} \Omega$ and $L_{S}=3 \mathrm{mH}$. G, power grid with parameters: $R_{S}=30 \mathrm{~m} \Omega$ and $L_{S}=1 \mathrm{mH}$. $H$, power grid with parameters: $R_{S}=30 \mathrm{~m} \Omega$ and $L_{S}=1 \mathrm{mH}$ with limitation $\varepsilon(5) \geq 0.45$. I, power grid with parameters: $R_{S}=30 \mathrm{~m} \Omega$ and $L_{S}=1 \mathrm{mH}$ with limitation $\varepsilon(5) \geq 0.50$.

coefficient $M_{2}$ in these cases decreases. For the third filter, the optimum value of coefficient $M_{3}$ for grid $S_{1}$ is approximate as in Method B and for grid $S_{2}$ as in Method A; taking the growing limitation into account decreases coefficient $M_{3}$. In the case of the fourth filter, the optimum value of coefficient $M_{4}$ resembles Methods $\mathrm{C}$ and $\mathrm{D}$ and decreases with an increase in the limitation for the first filter. A decrease in the grid equivalent impedance causes a decrease in reactive power attributed to the first filter and an increase in reactive power of the remaining filters.

Figure 3 presents graphs of reduction coefficient $\varepsilon_{S}$ for different design methods and for optimum methods in the function of the power grid equivalent inductance. There are enlarged characteristics' fragments which confirm that, for different grid impedances, different methods are more effective and that none of them is optimum. 


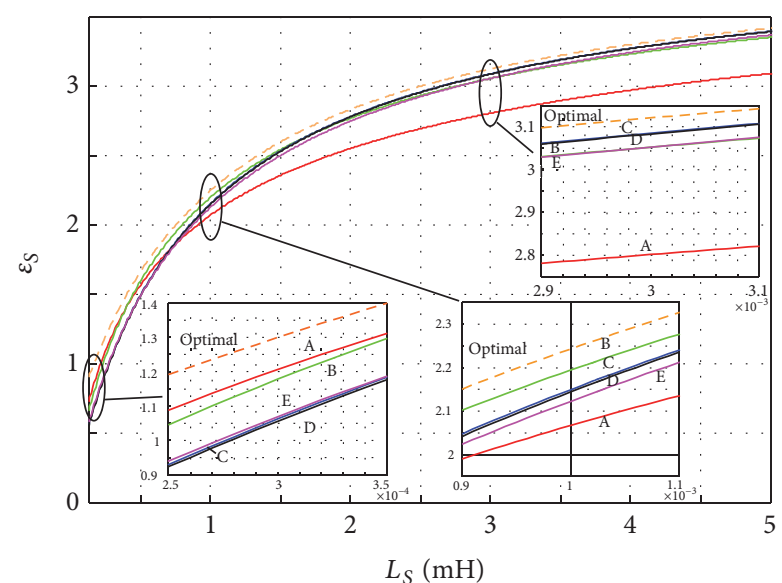

FIgURE 3: Characteristics of quality coefficient $\varepsilon_{S}$ for different methods of designing the filter group and for the optimum method in the function of the power grid equivalent inductance.

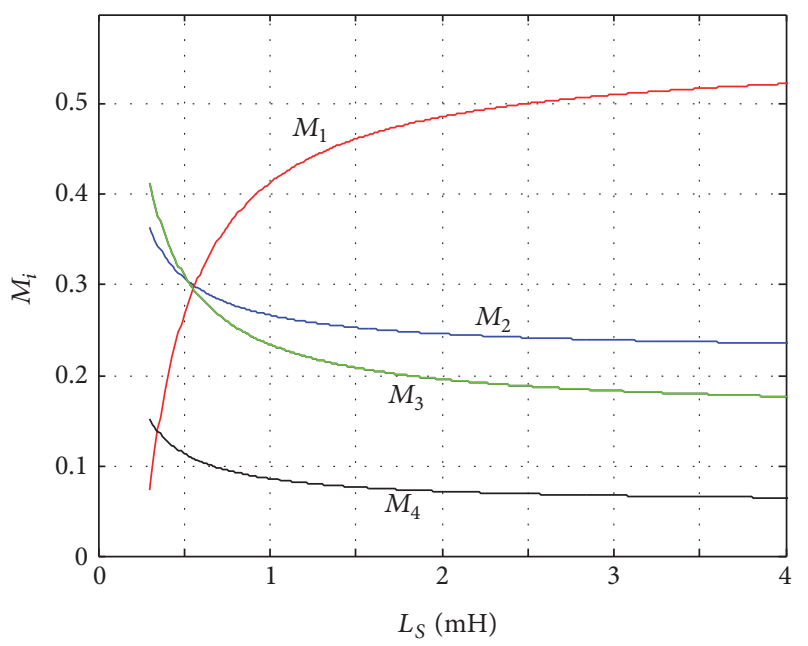

FIGURE 4: Graphs of optimum coefficients of reactive power distribution $M_{i}$ in the function of equivalent inductance $L_{S}$.

Figure 4 presents graphs of optimum coefficients of reactive power distribution $M_{i}$ in the function of the power grid equivalent inductance. Along with an increase in inductance, the share of the first filter's reactive power increases. It is related to a greater difficulty in reducing the lower-order harmonics in comparison to higher-order harmonics. Shares of the remaining filters decrease along with an increase in equivalent inductance.

The method of the impedance characteristics' maxima distribution is a method of designing the filter group equivalent to methods of reactive power distribution. Figure 5 presents the shift of these maxima (optimum) in the direction of higher harmonics along with the increase in the grid equivalent inductance. In the analysis of this graph, it must be remembered that the group's filter design assumes the filters' detuning from the eliminated harmonics. For low grid inductances, the location of the first maximum decreases below pulsation of the first of the eliminated harmonics (below the

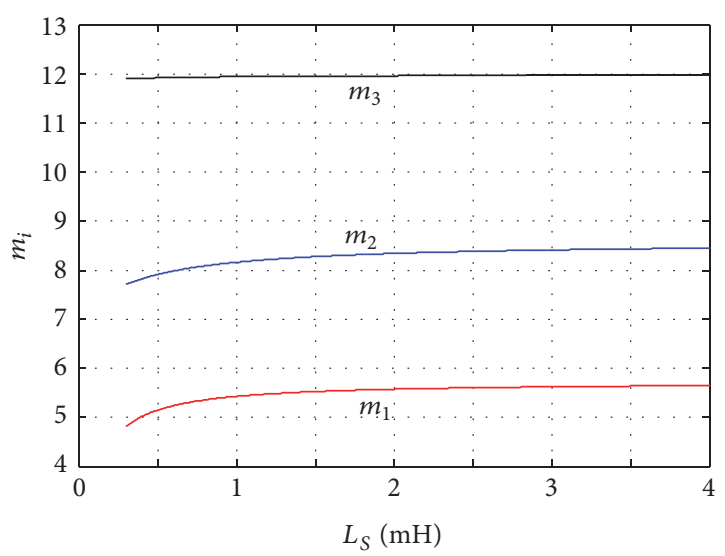

FIGURE 5: Graphs of optimum distributions of the filter group's impedance characteristics' maxima in the function of the power grid equivalent inductance.

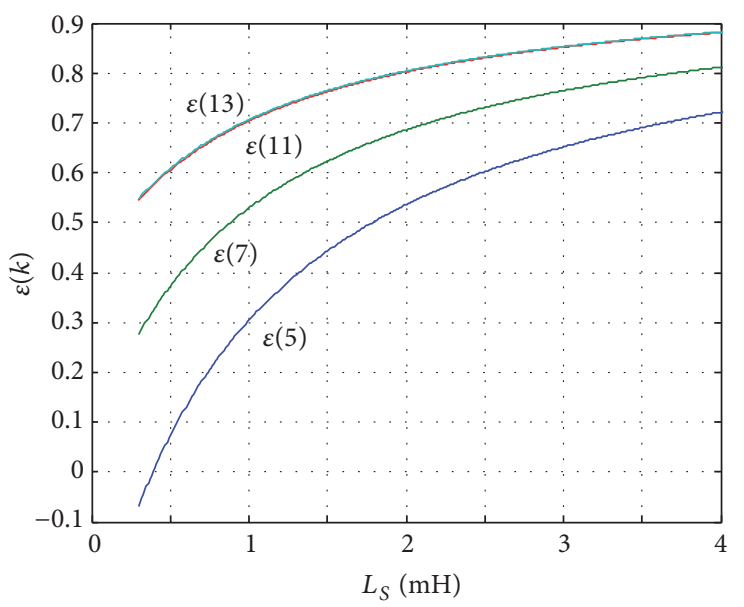

FIGURE 6: The reduction of selected harmonics by the optimum filter group in the function of the power grid equivalent inductance.

fifth harmonic). This means that this harmonic is amplified. Such a situation requires verifying the design assumptions in order to avoid harmonic amplification or at least ensuring that the amplification does not cause exceedances of values indicated in standards.

The graph in Figure 6 presents the increase in the reduction of selected harmonics along with the increase in the power grid equivalent inductance. The first graph of reduced harmonics (the fifth harmonics $\varepsilon(5)$ ) in the initial part has negative values, which indicates the amplification of this harmonic.

\section{Summary}

The article presents a uniform method of designing the simple filter group based on the determination of reactive power distribution between separate filters in the group. In the analysis, typically encountered methods of designing filter groups and the matrix method, based on the assumption of the location of impedance characteristics' resultant maxima, are 
taken into account. Uniform equations to determine the value of the filters' capacitance, inductance, resistance, and power, which all depend on the design assumptions and reactive power distribution, are presented. Formulas enabling quick conversion of filter parameters between design methods are specified. Also, a quick calculation of the filters' impedances and their real and imaginary resultants is possible. In the analysis, the filters' detuning from the reduced harmonics and quality factor of passive elements having effect on the filtration effectiveness are also taken into account. The important element affecting the filtration effectiveness is the power grid equivalent impedance, which was taken into account in the presented analysis. A criterion for evaluating the filter group's filtration effectiveness was proposed.

A best method cannot be chosen of the classical design methods because the filtration effectiveness depends strongly on the power grid equivalent impedance.

The optimum reactive power distribution for the filter group, according to the proposed criterion, determines the increase in the share of the first filter's reactive power along with the increase in the power grid equivalent impedance and the decrease in coefficients $M_{i}$ for the remaining filters.

The locations of the filter group's impedance characteristics' resultant maxima for the optimum reactive power distribution shift in the direction of higher frequencies along with the increase in the power grid equivalent impedance are discussed.

The filtration effectiveness increases with the increase in the grid impedance. For the low-power grid equivalent impedances, there is a risk of the low-order harmonics being amplified with the use of the proposed optimisation criterion.

Methods searching for reactive power distribution and the method of impedance characteristics' maxima location are equivalent methods of design.

The article does not discuss many practical issues of designing filters, for example, failure of one of the filters. The article was limited to the issue of designing the filter group through reactive power distribution between filter branches and taking into account the detuning of filters and their resistance.

\section{Competing Interests}

The author declares that there are no competing interests.

\section{References}

[1] B. Badrzadeh, K. S. Smith, and R. C. Wilson, "Designing passive harmonic filters for an aluminum smelting plant," IEEE Transactions on Industry Applications, vol. 47, no. 2, pp. 973-983, 2011.

[2] A. Lange and M. Pasko, "Selected problems of power energy quality in coal mines and steelworks," Przeglad Elektrotechniczny, vol. 88, no. 6, pp. 150-153, 2012.

[3] A. Lange and M. Pasko, "Reactive power compensation and filtration of higher harmonics generated to the network by arc furnaces," Przeglad Elektrotechniczny, vol. 88, no. 10, pp. 15-18, 2012.
[4] A. Lange and M. Pasko, "Compensation of the reactive power and filtration of high harmonics by means of passive LC filters," Przeglad Elektrotechniczny, vol. 86, no. 4, pp. 126-129, 2010.

[5] A. B. Nassif, W. Xu, and W. Freitas, "An investigation on the selection of filter topologies for passive filter applications," IEEE Transactions on Power Delivery, vol. 24, no. 3, pp. 1710-1718, 2009.

[6] C.-J. Chou, C.-W. Liu, J.-Y. Lee, and K.-D. Lee, "Optimal planning of large passive-harmonic-filters set at high voltage level," IEEE Transactions on Power Systems, vol. 15, no. 1, pp. 433-441, 2000.

[7] "Voltage characteristics of electricity supplied by public electricity networks," EN 50160, 2014.

[8] D. E. Steeper and R. P. Stratford, "Reactive compensation and harmonic suppression for industrial power systems using thyristor converters," IEEE Transactions on Industry Applications, vol. 12, no. 3, pp. 232-254, 1976.

[9] R. Klempka, Z. Hanzelka, and Y. Varetsky, "Bank harmonic filters operation in power supply system-cases studies," in Power Quality Issues, A. Zobaa, Ed., pp. 201-230, InTech, Rijeka, Croatia, 2013.

[10] R. Klempka, "Designing a group of single-branch filters taking into account their mutual influence," Archives of Electrical Engineering, vol. 63, no. 1, pp. 81-91, 2014.

[11] L. S. Czarnecki, "Common and fixed-poles resonant harmonic filters," European Transactions on Electrical Power, vol. 8, no. 5, pp. 345-351, 1998.

[12] G. J. Wakileh, Power Systems Harmonics: Fundamentals, Analysis and Filter Design, Springer, New York, NY, USA, 2001.

[13] J. Arrillaga, D. A. Bradley, and P. S. Bodger, Power System Harmonics, John Wiley \& Sons, 1985.

[14] L. S. Czarnecki and H. L. Ginn III, "The effect of the design method on efficiency of resonant harmonic filters," IEEE Transactions on Power Delivery, vol. 20, no. 1, pp. 286-291, 2005.

[15] C. Kawann and A. E. Emanucl, "Passive shunt harmonic filters for low and medium voltage: a cost comparison study," IEEE Transactions on Power Systems, vol. 11, no. 4, pp. 1825-1831, 1996.

[16] H. L. Ginn III and L. S. Czarnecki, "An optimization based method for selection of resonant harmonic filter branch parameters," IEEE Transactions on Power Delivery, vol. 21, no. 3, pp. 1445-1451, 2006.

[17] S. Sakar, A. D. Karaoglan, M. E. Balci, S. H. E. Abdel Aleem, and A. F. Zobaa, "Optimal design of single-tuned passive filters using response surface methodology," in Proceedings of the International School on Nonsinusoidal Currents and Compensation (ISNCC '15), Łagów, Poland, June 2015.

[18] A. F. Zobaa, "The optimal passive filters to minimize voltage harmonic distortion at a load bus," IEEE Transactions on Power Delivery, vol. 20, no. 2, pp. 1592-1597, 2005.

[19] R. Klempka, "Reactive power filter design using genetic algorithm," Przeglad Elektrotechniczny, vol. 5, pp. 294-301, 2013.

[20] A. Menti, T. Zacharias, and J. Milias-Argitis, "Optimal sizing and limitations of passive filters in the presence of background harmonic distortion," Electrical Engineering, vol. 91, no. 2, pp. 89-100, 2009.

[21] Y.-M. Chen, "Passive filter design using genetic algorithms," IEEE Transactions on Industrial Electronics, vol. 50, no. 1, pp. 202-207, 2003.

[22] Y. Zhao, H. Y. Deng, J. H. Li, and D. Z. Xia, “Optimal planning of harmonic filters on distribution systems by chance constrained programming," Electric Power Systems Research, vol. 68, no. 2, pp. 149-156, 2004. 
[23] A. A. Abou El-Ela, S. Allam, and H. El-Arwash, "An optimal design of single tuned filter in distribution systems," Electric Power Systems Research, vol. 78, no. 6, pp. 967-974, 2008.

[24] G. W. Chang, S.-Y. Chu, and H.-L. Wang, "A new method of passive harmonic filter planning for controlling voltage distortion in a power system," IEEE Transactions on Power Delivery, vol. 21, no. 1, pp. 305-312, 2006. 


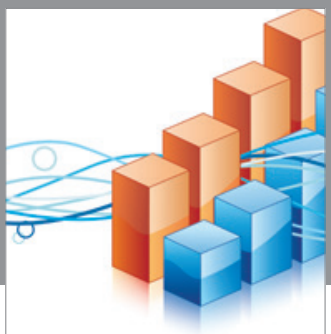

Advances in

Operations Research

vatem alat4

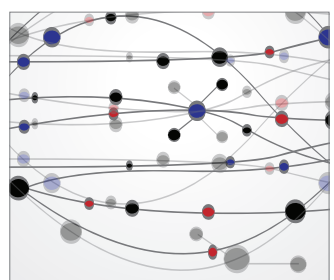

\section{The Scientific} World Journal
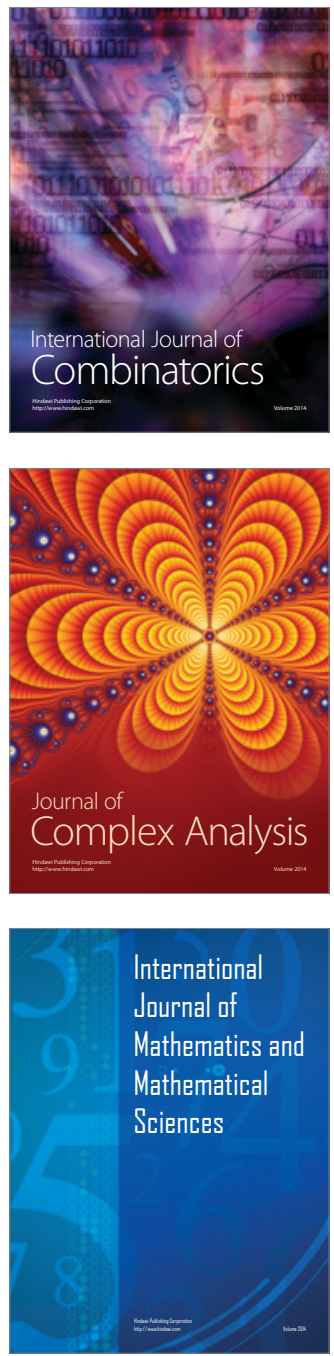
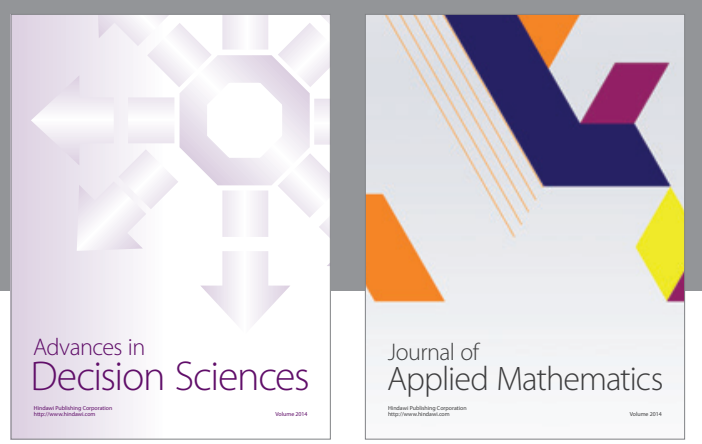

Algebra

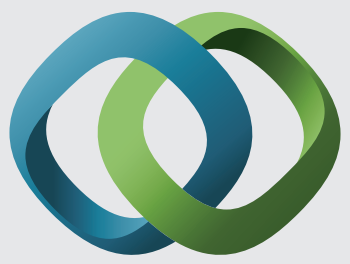

\section{Hindawi}

Submit your manuscripts at

http://www.hindawi.com
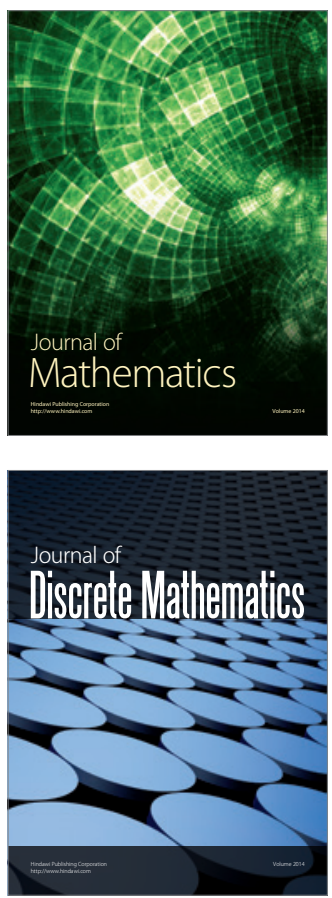

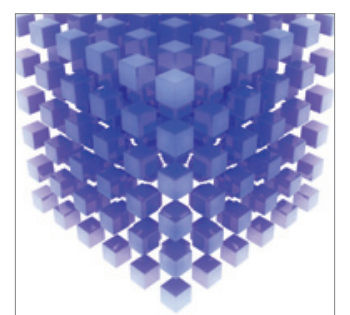

Mathematical Problems in Engineering
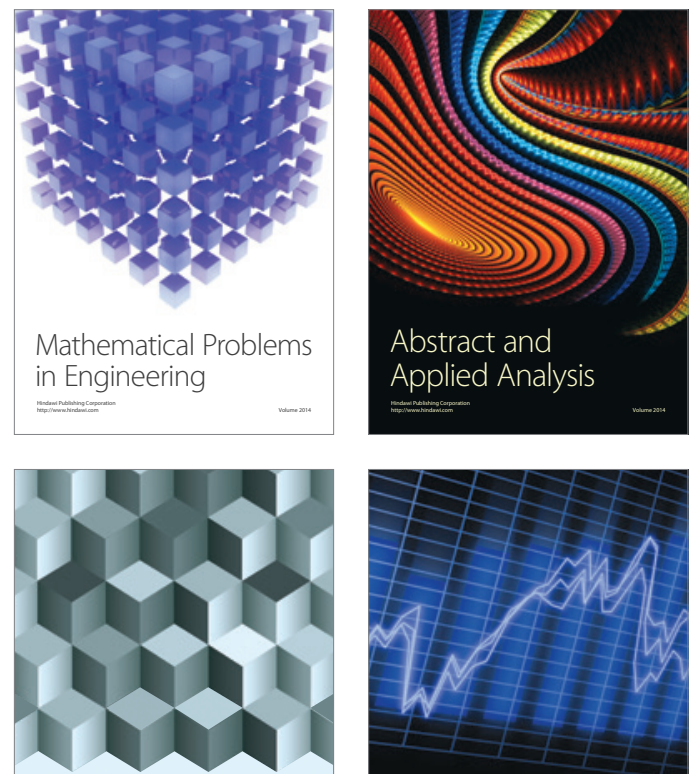

Journal of

Function Spaces

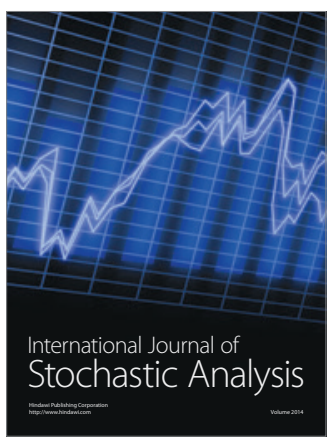

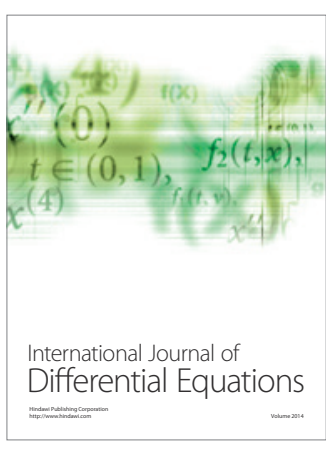
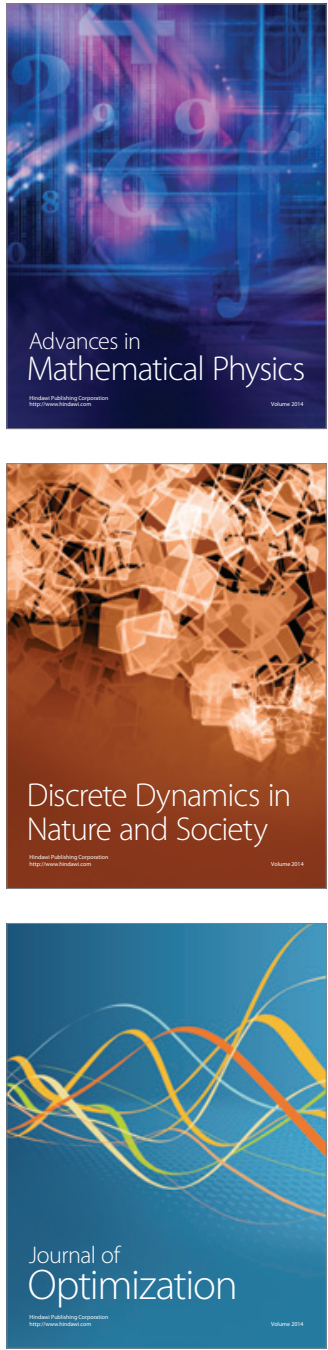